Hampshire, N. L., R. M. Mosier, T.M. Ryan, and D.E. Cavanaugh, 2018: Relationship of Low-Level Instability and Tornado Damage Rating Based on Observed Soundings. J. Operational Meteor., 6 (1), 1-12,

doi: https://doi.org/10.15191/nwajom.2018.0601

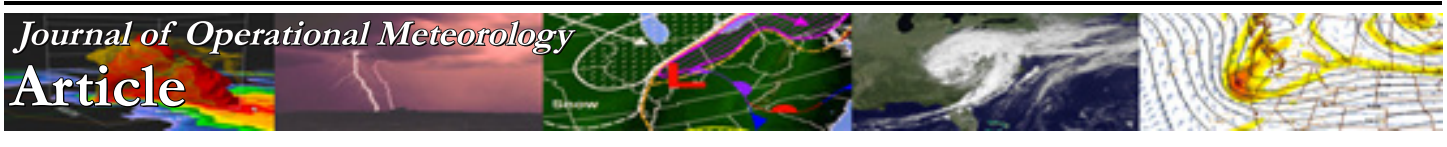

\title{
Relationship of Low-Level Instability and Tornado Damage Rating Based on Observed Soundings
}

\author{
NICHOLAS L. HAMPSHIRE \\ National Weather Service Weather Forecast Office, New Braunfels, Texas \\ RICHARD M. MOSIER \\ NOAA/NWS/NCEP Storm Prediction Center, Norman, Oklahoma \\ TED M. RYAN \\ National Weather Service Weather Forecast Office, Fort Worth, Texas \\ DENNIS E. CAVANAUGH \\ National Weather Service Weather Forecast Office, Little Rock, Arkansas
}

(Manuscript received 24 October 2017; review completed 18 January 2018)

\begin{abstract}
A database of upper-air soundings was collected for weak (EF0/EF1), significant (EF2/EF3), and violent (EF4/ EF5) tornadoes that occurred within $100 \mathrm{~km}$ and $6 \mathrm{~h}$ of the rawindsonde observation. After case filtering and quality control, a total of 50 proximity soundings for violent tornadoes and randomized samples of 100 proximity soundings for significant tornadoes and $\mathbf{1 0 2}$ for weak tornadoes were obtained. Key convective parameters were analyzed between the tornado datasets. Low-level instability parameters $(0-3-\mathrm{km}$ lapse rates and $0-3-\mathrm{km}$ mixed-layer convective available potential energy) were noteworthy predictors of the highest tornado damage rating, whereas mixed-layer lifted condensation level, wind shear, and effective storm relative helicity displayed little predictive skill distinguishing significant and violent tornado environments. The ability of the significant tornado parameter (STP) to discriminate between significant and violent tornadoes also was analyzed. This analysis found that STP does statistically discriminate between violent and significant tornadoes, with mixedlayer convective available potential energy the best discriminator of its variables. Because of the skill in the lowlevel instability parameters, this study also offers a new violent tornado parameter that includes the low-level instability fields in order to better differentiate between significant and violent tornado environments.
\end{abstract}

\section{Introduction}

Studies by Thompson et al. (2003, 2012), Togstad et al. (2011), Grams et al. (2012), and others have evaluated parameters to discriminate atmospheric conditions associated with higher probabilities of significant tornadoes (a magnitude of EF2 and higher) versus non-significant tornadoes. Less research has been conducted regarding parameters that distinguish environmental conditions specifically favorable for violent tornadoes (EF4 and EF5). Although violent tornadoes account for less than $1 \%$ of all reported tornadoes (McCarthy and Schaefer 2004), they are responsible for $66 \%$ of all tornado fatalities (Ashley 2007). Even though the number of violent tornadoes is relatively small, the environments associated with this tornado subset are worth investigating to determine if a discriminating signal exists to assist operational meteorologists in forecasting these rare events.

Thompson et al. (2003) used Rapid Update Cycle (RUC-2) proximity soundings to analyze supercell environments where significant tornadoes were reported

Corresponding author address: Nicholas Hampshire, NWS Austin/San Antonio TX, 2090 Airport Road, New Braunfels, TX 78130 E-mail: nick.hampshire@noaa.gov 
and found that composite parameters [specifically the significant tornado parameter (STP)] showed the best ability to distinguish between the two types. However, this study examined all EF2 or greater tornadoes, and the resulting statistics were likely weighted toward environments of EF2 and EF3 tornadoes because of the relative rarity of EF4 and EF5 tornadoes. Grams et al. (2012) studied a large sample of significant tornadoes and found that composite and kinematic parameters provided better discrimination versus thermodynamic parameters when forecasting significant tornado events, but again, any signal from the EF4 and EF5 tornadoes was overwhelmed by the relatively higher number of EF2 and EF3 tornadoes. Cohen (2010) used the same database of RUC-2 proximity soundings as Thompson et al. (2003) and concentrated on violent tornadoes but focused only on low-level kinematic, deep layer instability, and composite parameters similar to Thompson et al. (2003). The specific effects of low-level instability were not presented. Smith et al. (2015) did address violent tornadoes independently in terms of the STP and found values about two quartiles larger than the EF2 tornadoes. However, STP was the only parameter studied when investigating the violent tornado environments.

Davies (2002) found that low-level instability may play an important role in tornadogenesis occurring in atypical supercell tornado environments. Davies (2002) stated that significant tornadoes (EF2+) that develop in environments with relatively weak shear typically do so when there is sizable convective available potential energy (CAPE) in the low-levels of the atmosphere (0-3 km). Davies (2006) subsequently investigated tornadoes that occurred in environments with high lifted condensation level (LCL) heights. This study found that adequate low-level moisture and low-level CAPE in the presence of steep low-level lapse rates could help explain tornadogenesis in high LCL environments. Davies posits that low-level instability may have some effect on tornadogenesis and possibly EF-scale rating.

Markowski and Richardson (2010) stated that, once a tornado occurs, tilting of the surface-layer horizontal vorticity by the extreme vertical velocity gradient associated with the tornado updraft itself likely contributes to the near-ground vertical vorticity in a significant way. This suggests that as long as the environment remains supportive of extreme vertical velocities in the vicinity of the tornado updraft, the tornado should be able to persist. In addition, vertical motions in general are more responsive in environments characterized by low static stability (Nowotarski 2011). Many studies also have found low-level moisture to be a good indicator of an environment capable of producing a tornado (Rasmussen and Blanchard 1998; Grams et al. 2012).

By examining the potential relationship between low-level instability and the severity of the tornado damage rating, we suggest that an association exists between the two. In addition, this study also examines other kinematic and thermodynamic parameters to identify environments that are associated with violent tornadoes. Because of the infrequent occurrence of violent tornadoes, research that combined all EF2+ tornadoes together caused any EF4 and EF5 tornado environmental signals to be overwhelmed. For example, for the 27 April 2011 tornado outbreak, there were 15 violent tornadoes but 184 non-violent tornadoes (Knupp et al. 2014). Therefore, this study classifies cases in the following way: violent tornadoes (EF4 and EF5), significant tornadoes (EF2 and EF3), and weak tornadoes (EF0 and EF1).

\section{Data and methods}

Prior studies have used observed proximity soundings to assess near-storm environments when investigating thermodynamic and kinematic parameters associated with severe weather (Rasmussen and Blanchard 1998; Evans and Doswell 2001; Craven and Brooks 2004). These studies used different spatial criteria for proximity soundings ranging from 80 $\mathrm{km}$ (Brooks et al. 1994) to $400 \mathrm{~km}$ (Rasmussen and Blanchard 1998). This study selected $100 \mathrm{~km}$ as the spatial threshold for a near-optimal representation of the near-storm environment (Potvin et al. 2010). The temporal range in previous studies has been within 1 h (Brooks et al. 1994), 2 h (Evans and Doswell 2001), and $3 \mathrm{~h}$ (Craven and Brooks, 2004). For this study, any tornado within $6 \mathrm{~h}$ of the observational sounding was used because of the difficulties associated with obtaining soundings for the rare event of a violent tornado and the desire to get a reasonably large dataset. Figure 1 is a histogram that represents each tornado case and its time from the acquired sounding. This shows that $72 \%$ of the soundings used occurred within $3 \mathrm{~h}$ of the observed sounding time.

Fuhrmann et al (2014) noted there had been a substantial decrease in the number of significant and violent tornadoes coincident with a shift in responsibility for reporting tornadoes from the state 


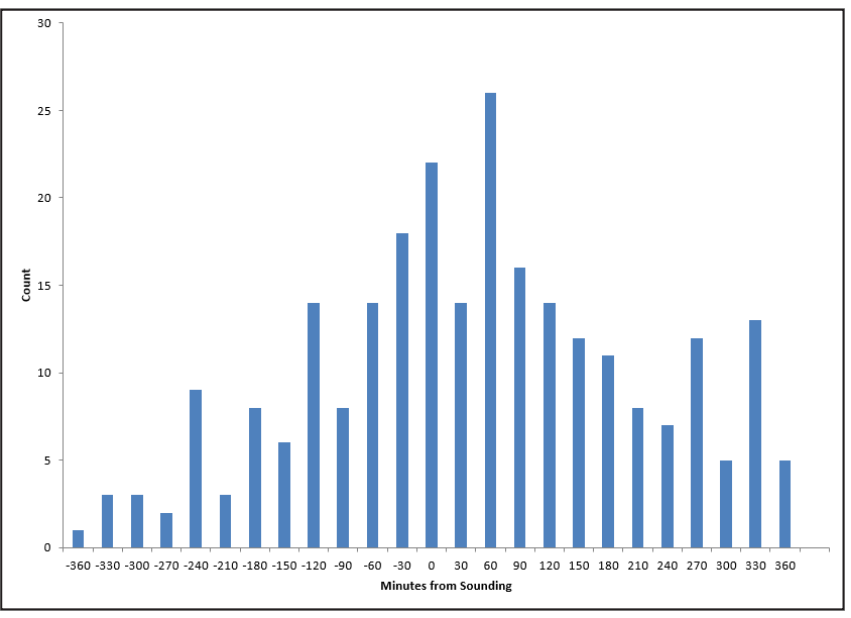

Figure 1. Histogram representing each case used in the study and its time from the observed proximity sounding. Click image for an external version; this applies to all tables and figures hereafter.

climatologists to the National Weather Service in 1973. Therefore, 1973 was chosen as the start date for this study to limit the influence of over-reported significant and violent tornadoes. The term "EF" is used for the damage ratings for all tornadoes, including those rated prior to the enhanced Fujita scale's implementation in 2007 (Edwards et al. 2012). The National Centers for Environmental Information publication, Storm Data, was examined from 1973-2015, and a database of significant and violent tornadoes was obtained for review. A database of weak tornadoes was only obtained from 2013-2015 owing to the vast number of cases available. To eliminate rawindsonde observations where the environment was not supportive of tornadogenesis, the following checks were implemented before a proximity sounding was allowed into the database: mixed-layer CAPE (MLCAPE) $>0 \mathrm{~J} \mathrm{~kg}^{-1}$, surfacebased CAPE $>0 \mathrm{~J} \mathrm{~kg}^{-1}, 0-3-\mathrm{km}$ storm-relative helicity $(\mathrm{SRH})>0 \mathrm{~m}^{2} \mathrm{~s}^{-2}, 0-1-\mathrm{km}$ SRH $>0 \mathrm{~m}^{2} \mathrm{~s}^{-2}$, and mixedlayer LCL (MLLCL) $<2000 \mathrm{~m}$. These spatial, temporal, and environmental criteria yielded a database of 50 violent tornadoes (EF4+), 1841 significant tornadoes (EF2/EF3), and 314 weak tornadoes (EF0/EF1). Instead of investigating all significant and weak tornadoes, a randomized sample was generated to compile 100 cases for significant tornadoes and 102 cases for weak tornadoes. In addition, a proximity sounding was used only once for its highest rating if it was repeated in a different severity dataset. Weaker tornadoes often occur near violent tornadoes (Knupp et al. 2014) and would be represented by the same near-storm environment, but the intent of this study is to best document the highest tornado potential sampled by the observed proximity sounding.

Once the data were obtained for review, statistical testing was conducted on each parameter or variable to determine if the findings were statistically significant. To accomplish this, several tests were run that included the non-parametric Mann-Whitney, two-tailed MannWhitney, Kruskal-Wallis, post-hoc Mann-Whitney, and a logistic regression test (Albright and Winston 2014).

\section{Results}

\section{a. Previously researched parameters}

Thompson et al. (2003) and Grams et al. (2012) found that composite parameters such as STP do best in distinguishing between significant tornado environments and non-tornadic environments. The authors opted to use the latest update for the STP (Thompson et al. 2012), which uses effective shear (EBWD) and effective storm-relative helicity (ESRH) and incorporates mixed-layer convective inhibition (MLCIN). The formula for the STP is as follows:

$\mathrm{STP}=\left(\mathrm{MLCAPE} / 1500 \mathrm{~J} \mathrm{~kg}^{-1}\right) *\left(\mathrm{EBWD} / 20 \mathrm{~m} \mathrm{~s}^{-1}\right) *$ $\left(\mathrm{ESRH} / 150 \mathrm{~m}^{-2} \mathrm{~s}^{-2}\right) *((2000-\mathrm{MLLCL}) / 1000 \mathrm{~m}) *$ $\left((200+\right.$ MLCIN $\left.) / 150 \mathrm{~J} \mathrm{~kg}^{-1}\right)$

The EBWD term is capped at a value of 1.5 and the $\mathrm{STP}$ is 0 if the value is less than $12 \mathrm{~m} \mathrm{~s}^{-1}$. The MLLCL term is set to 1 for MLLCL heights $<1000 \mathrm{~m} \mathrm{AGL}$ and to 0 for MLLCL heights $>2000 \mathrm{~m} \mathrm{AGL}$, and the MLCIN term is set to 1 for MLCIN values $>-50 \mathrm{~J} \mathrm{~kg}^{-1}$ and to 0 for MLCIN $<-200 \mathrm{~J} \mathrm{~kg}^{-1}$.

The analysis of the STP from this tornado dataset (Fig. 2) showed the mean value increased from 0.54 to 1.22 to 3.03 , and the medians increased from 0.28 to 0.85 to 2.33 for the weak, significant, and violent tornado datasets, respectively. Also, the median of the violent cases exceeded the 75th percentile of significant cases, and the 75th percentile of violent cases exceeded the 90th percentile of significant cases.

To determine whether the STP was statistically equivalent for the significant and violent tornado environments sampled, a hypothesis test called the non-parametric Mann-Whitney test was performed to limit assumptions about the distributions of the data. A two-tailed Mann-Whitney test was run for the STP data collected for significant versus violent tornado 


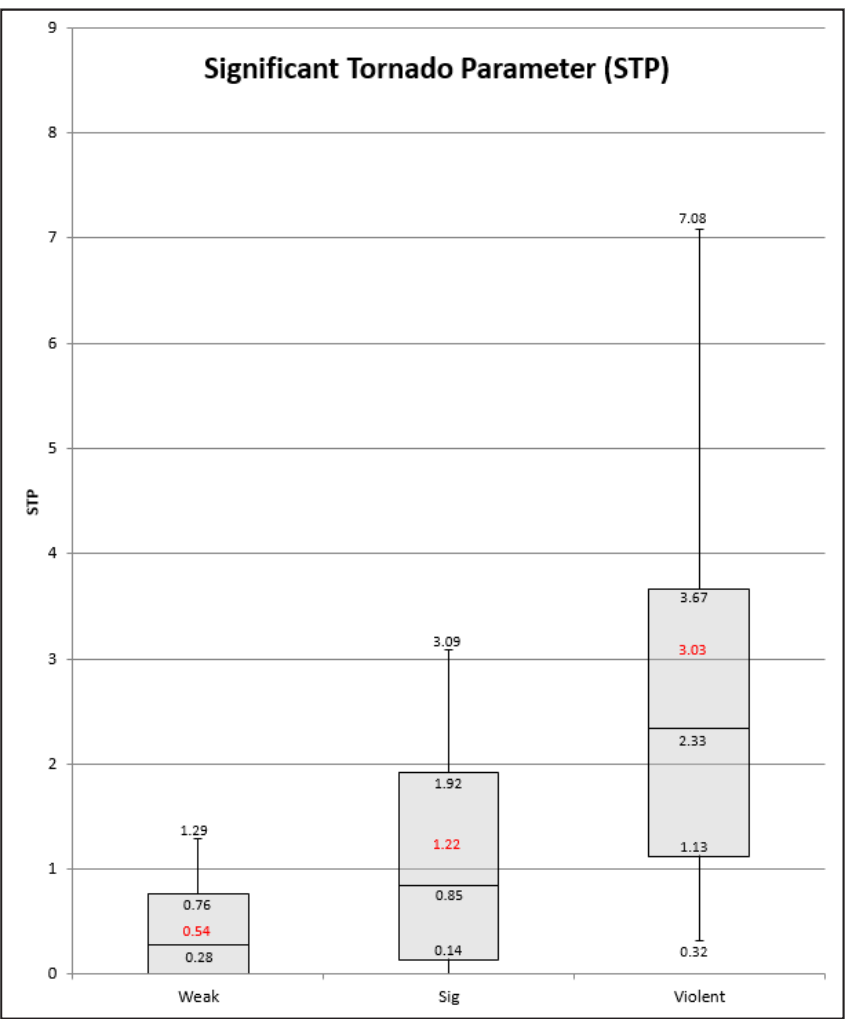

Figure 2. Box and whisker plots for the STP for weak, significant, and violent tornado environments. Values in black represent the 10th, 25th, 50th, 75th, and 90th percentile values from bottom to top. If there are no corresponding lines for the respective values, the value represented is zero. The mean value is in red. The sample consisted of 102 weak, 100 significant, and 50 violent tornadoes.

environments with the null hypothesis stating STP should be equal for these two environments. The alternative hypothesis stated either distribution may be smaller than the other. The resultant test statistic scored a -4.4 , providing strong evidence that this null hypothesis should be rejected. The p-value corresponding to this test statistic was $<0.0001$, indicating that the null hypothesis can be rejected at the $0.01 \%$ significance level (or $99.99 \%$ confidence level). In addition, the results of the Mann-Whitney test indicated the STP associated with violent tornadoes was greater than the STP associated with significant tornadoes. These results are similar to the findings from Smith et al. (2015).

In addition to the Mann-Whitney test comparing just the significant and violent tornado environments, a Kruskal-Wallis test compared the statistical distributions of all three tornado categories to determine if the distributions are the same. The results of this test confirmed the results of the Mann-Whitney test by once again rejecting the null hypothesis of equivalent distributions in each category with an associated p-value of $<0.0001$. Post-hoc Mann-Whitney tests (using a Bonferroni correction) also were performed to compare each pair of tornado damage rating categories, and each pairing was shown to reject the null hypothesis of equivalent distributions with associated $\mathrm{p}$-values each $<0.0001$. These findings increased the confidence in the results of the individual Mann-Whitney test performed on only the significant and violent tornado environment STP values. Only individual Mann-Whitney test results are discussed hereafter in the text for brevity, as this test appears to be representative of other statistical hypothesis testing techniques.

With the STP shown to be beneficial in discriminating between tornado environments, the individual elements of the STP equation (Eq. 1) were investigated to see which component variables do best at identifying violent tornado cases. The mean values of MLCAPE increased from 947 to 1330 to $2233 \mathrm{~J} \mathrm{~kg}^{-1}$, and the medians increased from 803 to 999 to $2209 \mathrm{~J} \mathrm{~kg}^{-1}$ for the weak, significant, and violent tornado datasets, respectively (Fig. 3). Like the STP, the median of the violent cases exceeded the 75 th percentile of significant cases, and the 75th percentile of violent cases exceeded the 90th percentile of the significant cases. The MannWhitney test statistic for MLCAPE scored a -4.0 when computed for violent versus significant tornado environments. Once again, this provides strong evidence to reject the null hypothesis of equivalent distributions (p-value $<0.0001$ ) and indicates that MLCAPE values associated with violent tornado environments are greater than those associated with significant tornado environments.

The mean values of the EBWD (Fig. 4) increased from 20.0 to 24.1 to $27.4 \mathrm{~m} \mathrm{~s}^{-1}$, and the medians increased from 20.1 to 24.2 to $27.5 \mathrm{~m} \mathrm{~s}^{-1}$ for the weak, significant, and violent tornado cases, respectively. This parameter shows promise for distinguishing the tornado environments more favorable for significant tornadoes; however, the increases from the significant to the violent cases were not as notable as with MLCAPE or STP. The Mann-Whitney test statistic only scored a -1.9 when comparing the violent and significant tornado databases for the EBWD. This test statistic resulted in a p-value of 0.0523 , which fails to reject the null hypothesis at the $5 \%$ significance level. Thus, the EBWD distributions for significant and violent tornadoes cannot be considered different with high confidence. This test also indicates 


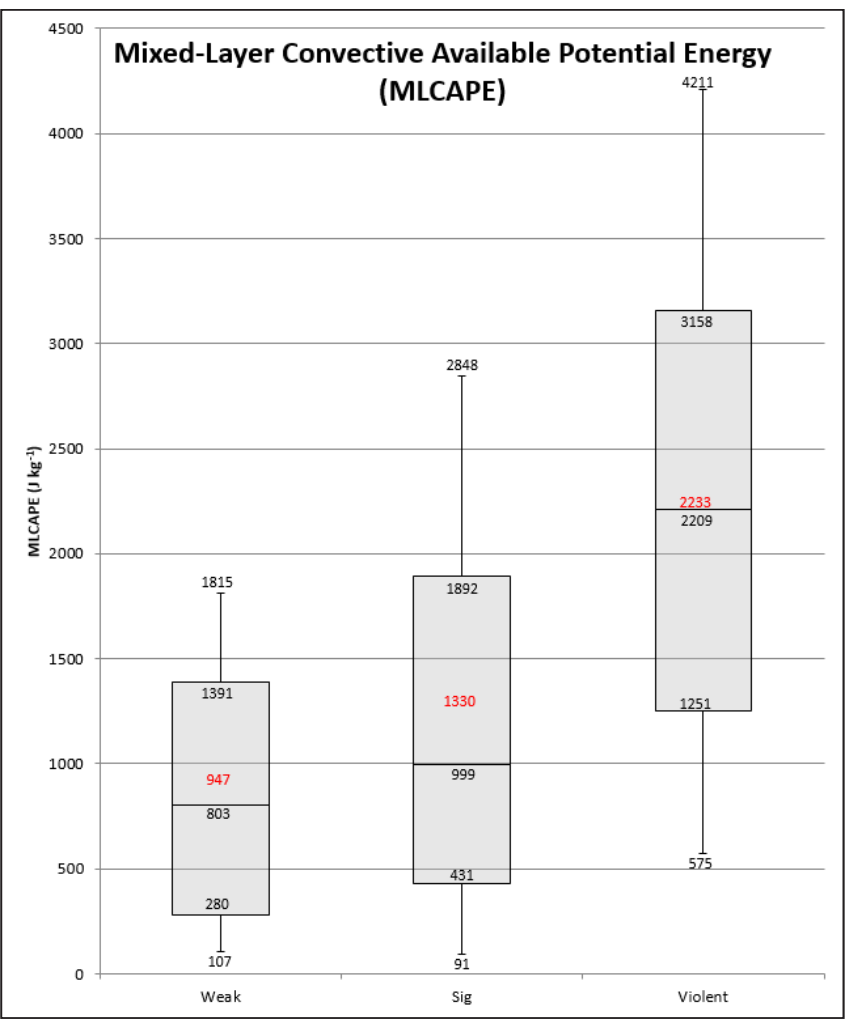

Figure 3. Same as Fig. 2 but for MLCAPE $(\mathrm{J} \mathrm{kg-1})$.

that there is quite a bit of overlap between the parameter spaces of these two tornado damage rating categories using this metric alone. Consequently, compared to MLCAPE and STP, EBWD does not show sufficient skill at distinguishing violent tornado from significant tornado environments.

Mean values of ESRH (Fig. 5) increased from 140 to 188 to $250 \mathrm{~m} 2 \mathrm{~s}^{-2}$, and the medians increased from 121 to 163 to $212 \mathrm{~m} 2 ~ s^{-2}$ for the weak, significant, and violent tornado cases, respectively. Although there were increases from the weak cases to significant cases and further increases from the significant cases to violent cases, these increases were not as notable as with STP and MLCAPE. The Mann-Whitney test statistic for ESRH scored a -2.4 for the comparison of the violent and significant tornado environments. This test statistic resulted in p-value of 0.0177 , so the null hypothesis is rejected at the 5\% significance level but fails to be rejected at the $1 \%$ significance level. Similar to the results of the EBWD test, this does not indicate that the distributions are equal, but provides evidence that there is enough overlap between these two datasets to result in less skill in discriminating the environments when compared to STP and MLCAPE.

Mean values of MLLCL (Fig. 6) decreased from

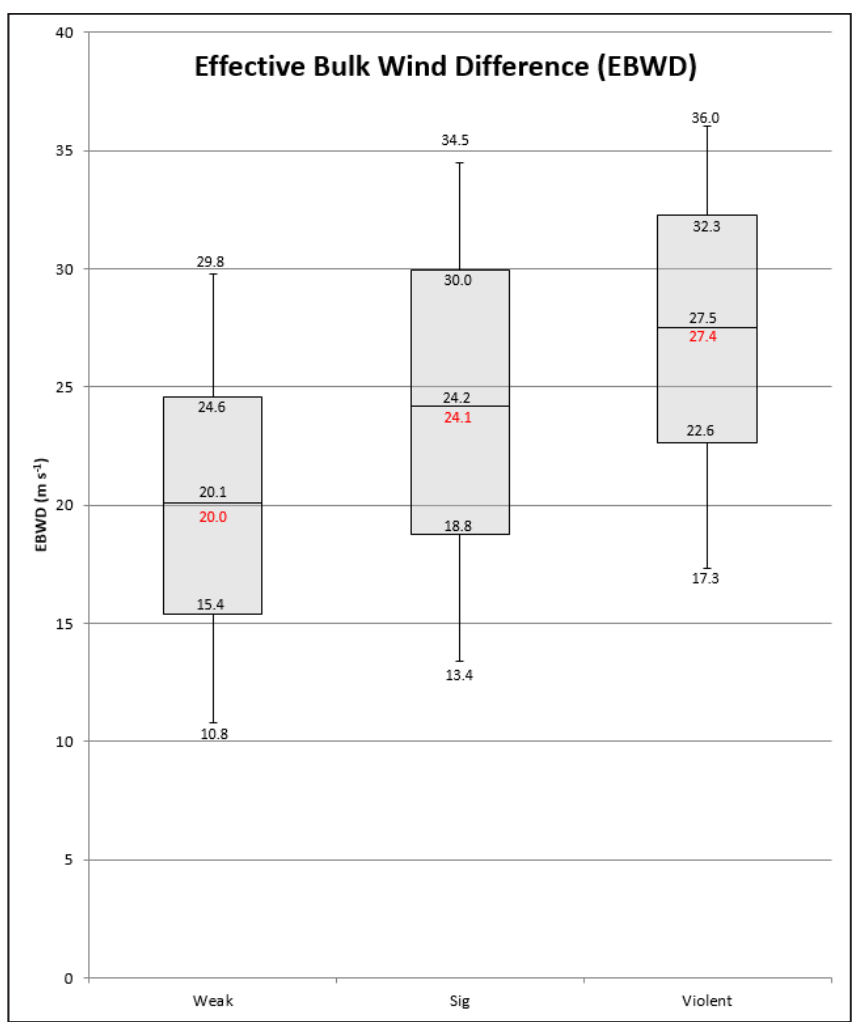

Figure 4. Same as Fig. 2 except for EBWD $\left(\mathrm{m} \mathrm{s}^{-1}\right)$.

$1017 \mathrm{~m}$ for the weak tornado dataset to $907 \mathrm{~m}$ for the significant dataset and slightly decreased to $897 \mathrm{~m}$ for the violent tornado dataset. However, the medians within the datasets fluctuated between 881, 833, and 894 meters for the weak, significant, and violent tornado datasets, respectively. The significant dataset had the lowest median value of MLLCL, which is what previous research has shown regarding LCL height and its relationship with tornado EF-scale rating (Thompson et al. 2003). However, the violent dataset was the highest value of the cases. The Mann-Whitney test statistic for MLLCL was -0.7 when comparing the violent and significant tornado environments. The corresponding p-value to this test was 0.4618 , which fails to reject the null hypothesis at all relevant significance levels. This test result indicates that the distribution of MLLCL associated with violent and significant tornado environments is essentially equivalent. There was no meaningful statistical separation of these categories for this parameter.

The MLCIN component (Fig. 7) showed similar results to the EBWD and ESRH in discriminating violent tornado environments. The mean values ranged from -43 to -56 to $-22 \mathrm{~J} \mathrm{~kg}^{-1}$, and the medians ranged from -27 to -25 , to $-17 \mathrm{~J} \mathrm{~kg}^{-1}$ for the weak, significant, 


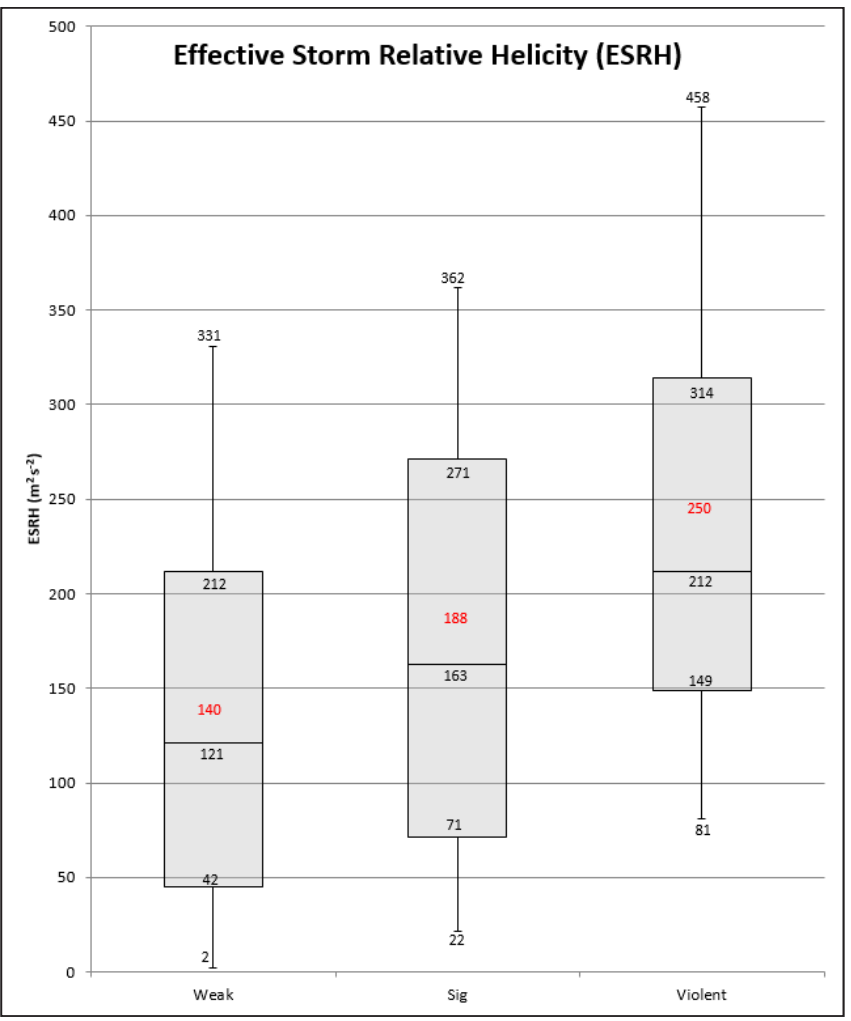

Figure 5. Same as Fig. 2 except for ESRH $\left(\mathrm{m}^{2} \mathrm{~s}^{-2}\right)$.

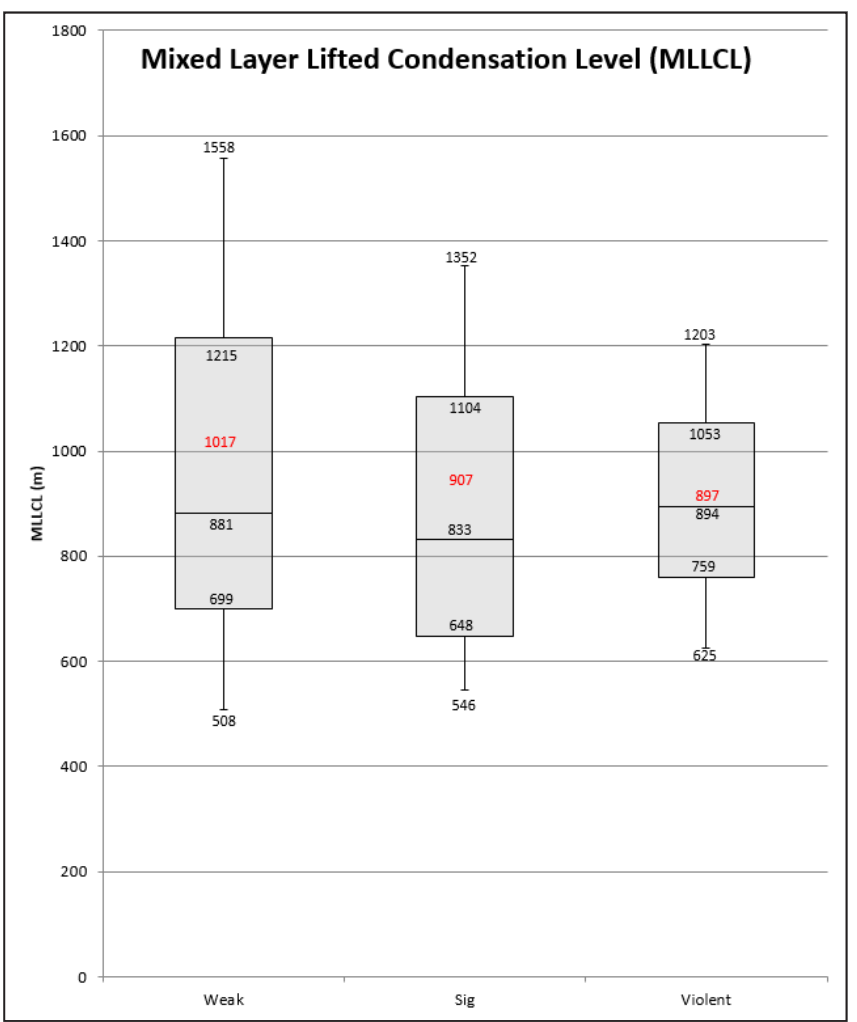

Figure 6. Same as Fig. 2 except for MLLCL (m).

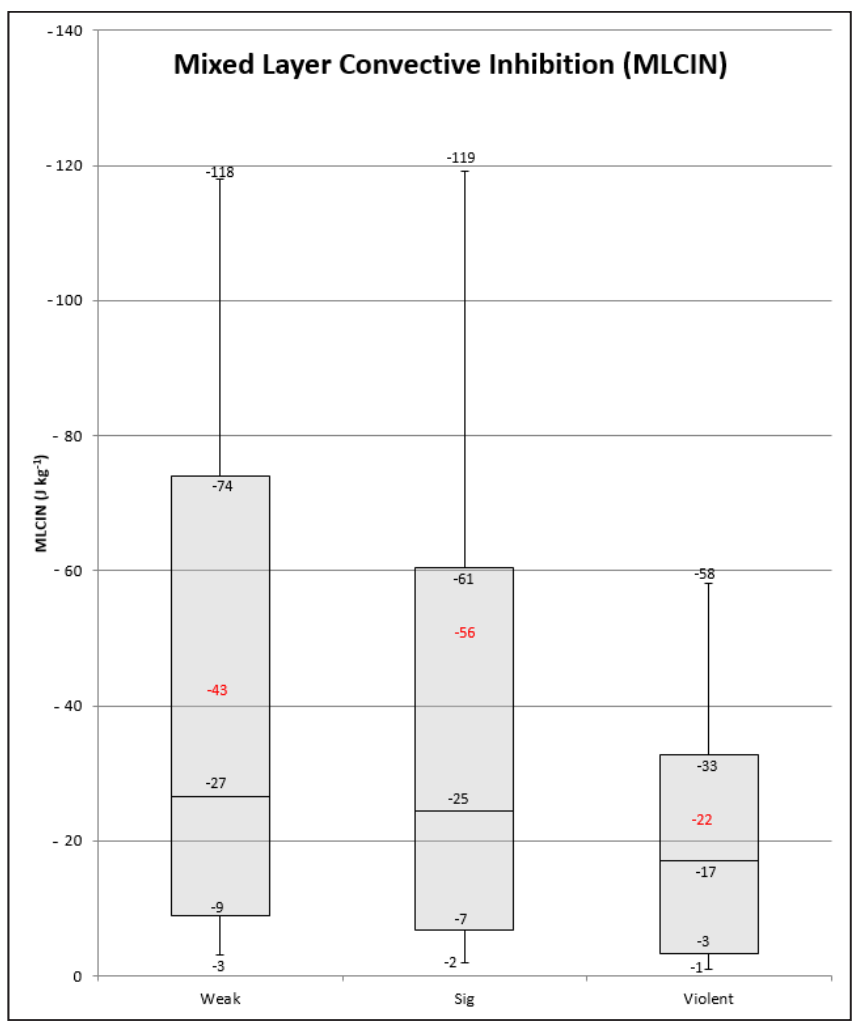

Figure 7. Same as Fig. 2 except for MLCIN $\left(\mathrm{J} \mathrm{kg}^{-1}\right)$. The y-axis is reversed with the largest to smallest values ascending from bottom to top.

and violent tornado databases, respectively. Although there were small increases in the median values as the tornado damage rating category increased, the MLCIN showed a weak signal in differentiating violent tornado versus significant tornado environments. The Mann-Whitney test statistic for MLCIN scored a -2.3 for the comparison of the violent and significant tornado environments. The corresponding $\mathrm{p}$-value here was 0.0220 , so the results are on par with the results of the EBWD and ESRH test results.

To summarize, the STP shows skill at discriminating between weak and significant tornado environments as shown by previous research but also helps to discriminate violent tornadoes. The MLCAPE component of the STP was shown to be best at discriminating violent tornado environments.

When ranking each parameter of the STP by its ability to distinguish significant versus violent tornado environments based on the Mann-Whitney tests (Table 1), MLCAPE is far better than the second-best parameter, ESRH. The other parameters (MLCIN, EBWD, and MLLCL) show only minimal utility in distinguishing violent tornado environments from 
significant tornado environments.

\section{b. Low-level instability parameters}

Because MLCAPE provided skill in distinguishing the significant tornado environments from the violent cases, it follows that low-level instability parameters also should be investigated. The 0-3-km MLCAPE (hereafter 3CAPE; Fig. 8) had a mean value for weak tornado cases of $52 \mathrm{~J} \mathrm{~kg}^{-1}$ with a slight increase to 66 $\mathrm{J} \mathrm{kg}^{-1}$ for significant tornadoes but jumped to $99 \mathrm{~J} \mathrm{~kg}^{-1}$ for violent tornadoes. The median values saw the same general trend with values rising from 38 to 50 to 95 $\mathrm{J} \mathrm{kg}^{-1}$ for the weak, significant, and violent tornado datasets, respectively. The Mann-Whitney test scored a -3.3 for the comparison of the violent and significant tornado databases for 3CAPE. The corresponding p-value for this test was 0.0009 , which supports rejecting the null hypothesis of equivalent distributions at the $1 \%$ significance level. This result provides evidence that 3CAPE associated with violent tornado environments is higher than that of significant tornado environments, and it shows more skill than the shearbased components of the STP in discriminating violent

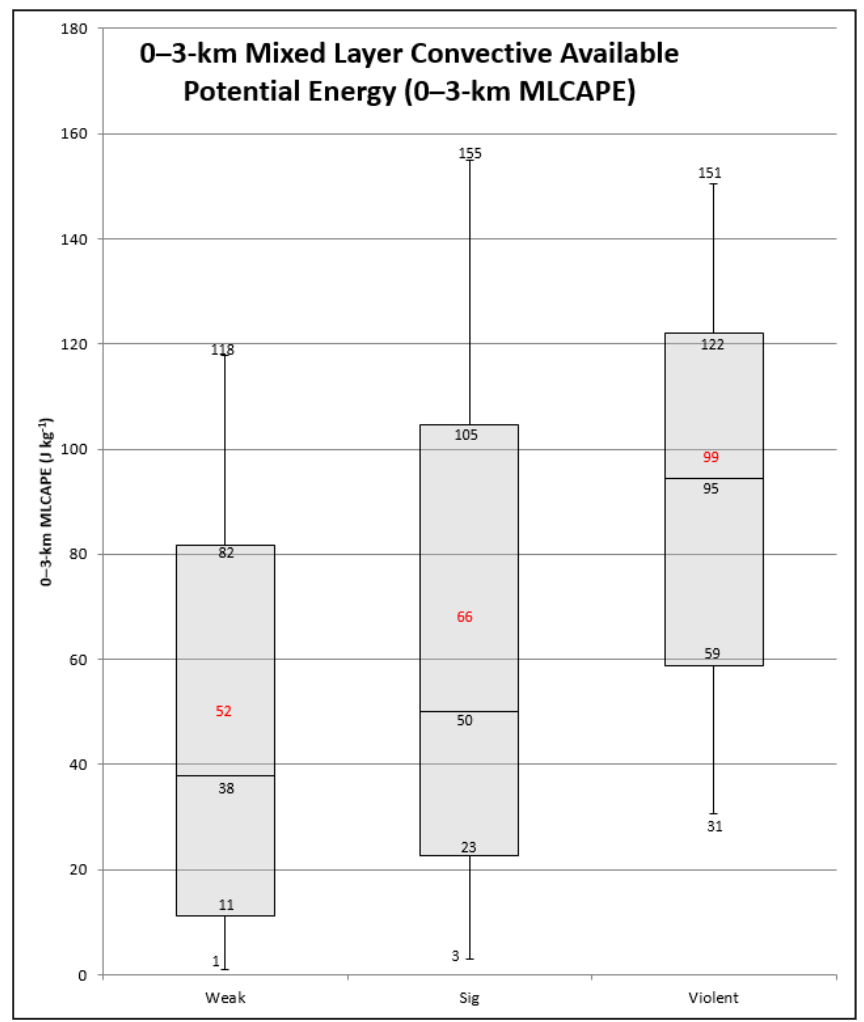

Figure 8. Same as Fig. 2 except for $0-3 \mathrm{~km}$ MLCAPE $\left(\mathrm{J} \mathrm{kg}^{-1}\right)$.

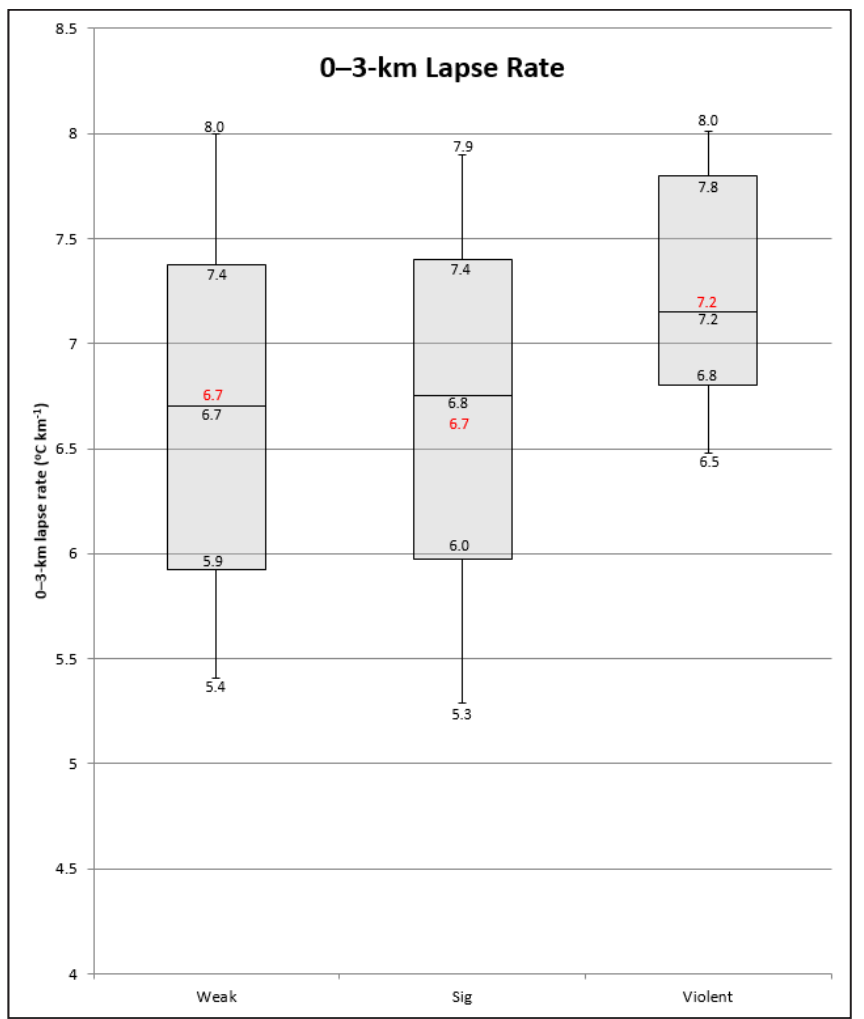

Figure 9. Same as Fig. 2 except for $0-3-\mathrm{km}$ lapse rate $\left({ }^{\circ} \mathrm{C} \mathrm{km}^{-1}\right)$.

from significant tornado environments.

The 0-3-km lapse rates (hereafter 3LR; Fig. 9) showed similar promise in comparison to the 3CAPE. The mean value for the significant tornadoes was equal to weak tornadoes with a value of $6.7^{\circ} \mathrm{C} \mathrm{km}^{-1}$, but there was an increase to $7.2{ }^{\circ} \mathrm{C} \mathrm{km}^{-1}$ for violent tornadoes. The median values had a slight increase from $6.7^{\circ} \mathrm{C}$ $\mathrm{km}^{-1}$ for weak tornadoes to $6.8{ }^{\circ} \mathrm{C} \mathrm{m}^{-1}$ for significant tornadoes to $7.2{ }^{\circ} \mathrm{C} \mathrm{km}^{-1}$ for violent tornadoes. In addition to the increases shown for violent tornadoes, the standard deviation of the violent dataset dropped to 0.77 compared with the 1.04 and 0.99 of the weak and significant databases. Less variability in the data suggests violent tornadoes rarely occur in environments with weaker low-level lapse rates. The Mann-Whitney test scored a -3.6 for the comparison of the violent and significant tornado databases for 3LR. This test statistic had a corresponding p-value of 0.0004 , which indicates a very similar result to the 3CAPE Mann-Whitney test and can be interpreted in a similar manner.

The Mann-Whitney test scores (Table 1) for both the 3LR and 3CAPE scored better than any other parameter incorporated into the STP other than the MLCAPE. Therefore, to help identify violent tornado 
Table 1. Mann-Whitney scores of the violent versus significant tornado databases of the parameters examined in this study.

\begin{tabular}{|c|c|}
\hline Parameter & Mann-Whitney Score (Violent versus Significant) \\
\hline STP & -4.4 \\
\hline MLCAPE & -4.0 \\
\hline EBWD & -1.9 \\
\hline ESRH & -2.4 \\
\hline MLLCL & -0.7 \\
\hline MLCIN & -2.3 \\
\hline \hline $0-3-k m$ CAPE & -3.3 \\
\hline 0-3-km Lapse Rate & -3.6 \\
\hline VTP & -5.3 \\
\hline
\end{tabular}

environments, these results suggest that it might be beneficial to incorporate either 3CAPE and/or 3LR into an amended version of the STP.

\section{c. Violent tornado parameter}

Given that low-level instability shows promise in helping discern violent tornado environments, the authors examined 3CAPE and 3LR to find thresholds or breakpoints in the data that could be used to better forecast violent tornado environments. These breakpoints were then included in the STP to help the composite parameter better distinguish violent tornado environments. Breakpoints were chosen after examining the median and percentile values for weak and violent tornadoes and testing how these values compared across all tornado intensities. The values chosen were $6.5{ }^{\circ} \mathrm{C} \mathrm{km}^{-1}$ for $3 \mathrm{LR}$ and $50 \mathrm{~J} \mathrm{~kg}^{-1}$ for 3CAPE. The value chosen for $3 \mathrm{LR}$ was $6.5^{\circ} \mathrm{C} \mathrm{km}^{-1}$ since it was the 10 th percentile value for the violent cases but also near the median of both the weak and significant datasets. The value chosen for 3CAPE was $50 \mathrm{~J} \mathrm{~kg}^{-1}$ because it represented the median of the significant tornado dataset but was also near the lowest quartile of the violent tornado cases.

Although the STP does do a good job at discriminating violent tornado environments, improvements could be made to the equation by adding low-level instability parameters (3CAPE and 3LR). The authors will refer to this amended STP equation as the violent tornado parameter (VTP) to provide easy comparisons to show its improvement in detecting violent tornado environments within our tornado dataset. The added factors were constructed in a way like those already in STP, using the breakpoints determined above as the normalization values.

$\mathrm{VTP}=\left(\mathrm{MLCAPE} / 1500 \mathrm{~J} \mathrm{~kg}^{-1}\right) *\left(\mathrm{EBWD} / 20 \mathrm{~m} \mathrm{~s}^{-1}\right)$ * (ESRH/150 $\left.\mathrm{m}^{-2} \mathrm{~s}^{-2}\right) *((2000-\mathrm{MLLCL}) / 1000 \mathrm{~m}) *$ $\left((200+\right.$ MLCIN $\left.) / 150 \mathrm{~J} \mathrm{~kg}^{-1}\right) *\left(3 \mathrm{CAPE} / 50 \mathrm{~J} \mathrm{~kg}^{-1}\right) *$ $\left(3 \mathrm{LR} / 6.5^{\circ} \mathrm{C} \mathrm{km}^{-1}\right)$

The same restrictions for the STP terms previously mentioned still apply for the VTP. The 3CAPE factor is capped at a value of 2 .

The VTP (Fig. 10) does show higher values than the traditional STP when comparing violent tornado to significant and weak tornado environments. Compared to the STP, the weak tornado database overall saw little change in the values. For the significant tornado database, VTP values up to the medians actually decreased compared to the STP with the median decreasing from 0.85 (STP) to 0.63 (VTP). However, the upper percentile values and the mean had slight increases. Greater changes were noted with the VTP in the violent tornado database compared to the STP. The 10th percentile increased from 0.32 to 0.41 , the 25 th percentile increased from 1.13 to 1.74 , the median increased from 2.33 to 3.46 , the 75 th percentile increased

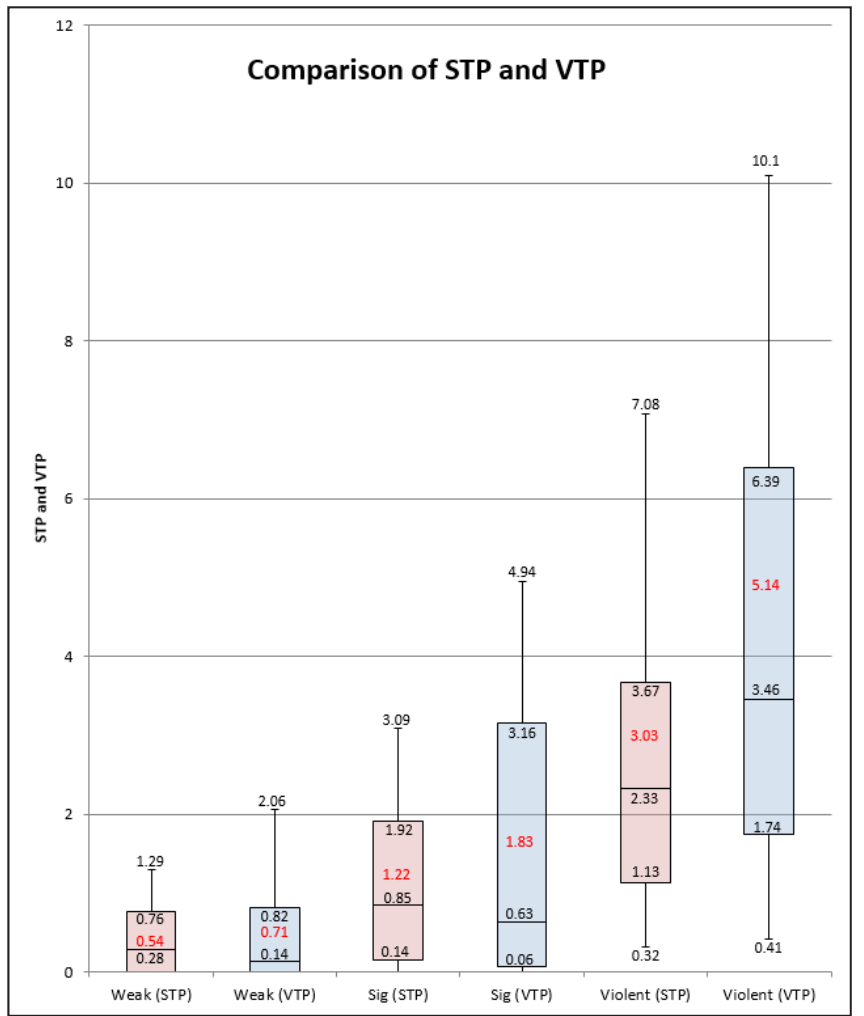

Figure 10. Same as Fig. 2 except for a comparison of the STP and VTP. 
from 3.67 to 6.39 , the 90th percentile increased from 7.08 to 10.1 , and the mean value increased from 3.03 to 5.14. The median STP had a violent to significant ratio of 2.7, while the VTP had a violent/significant ratio of 5.4. To summarize, there were negligible differences between the STP and VTP for the weak and significant tornado database, but substantial increases were noted for the violent tornadoes. The Mann-Whitney test scored a -5.3 when comparing the violent tornado and significant tornado databases for the VTP. This compares to the -4.4 of the STP. The corresponding p-value for both test statistics was $<0.0001$ and, as a result, it is difficult to determine (based on Mann-Whitney tests alone) which parameter is truly better at discriminating violent tornado environments from significant tornado environments.

A logistic regression test was performed on the STP and VTP to compare the ability of each parameter to properly identify an environment that favors a violent tornado when violent and significant tornado environments are combined in the same sample. A classification matrix was developed from the logistic regression equation, and this matrix showed that the STP had a $27 \%$ success rate when forecasting violent tornadoes compared to the VTP, which improved the success (hit) rate to $30 \%$. This shows that if a significant tornado environment is successfully identified, then the VTP offers some improvement when trying to identify those environments in which violent tornadoes will occur. Although the VTP shows a slight improvement in the ability to detect violent tornado environments, the STP still shows skill at identifying traditional EF2EF5 tornado environments compared to weak tornado environments.

To validate these results, a second randomized and independent subset of 100 significant and 100 weak tornado cases was obtained and tested against the 50 violent tornado cases to compare the results of STP and VTP previously discussed (Fig. 11). Because of the smaller sample size of violent tornadoes, a separate database of violent tornadoes could not be obtained. Once again, larger increases between the significant tornadoes and violent tornadoes were found for the VTP compared to the STP. The ratio of the median between violent and significant tornadoes increased from 2.8 for the STP to 4.8 for the VTP. This compares to the original dataset values of 2.7 and 5.4 for the STP and VTP, respectively. The same statistical testing was conducted for the STP and VTP comparing both parameters' ability to distinguish between significant

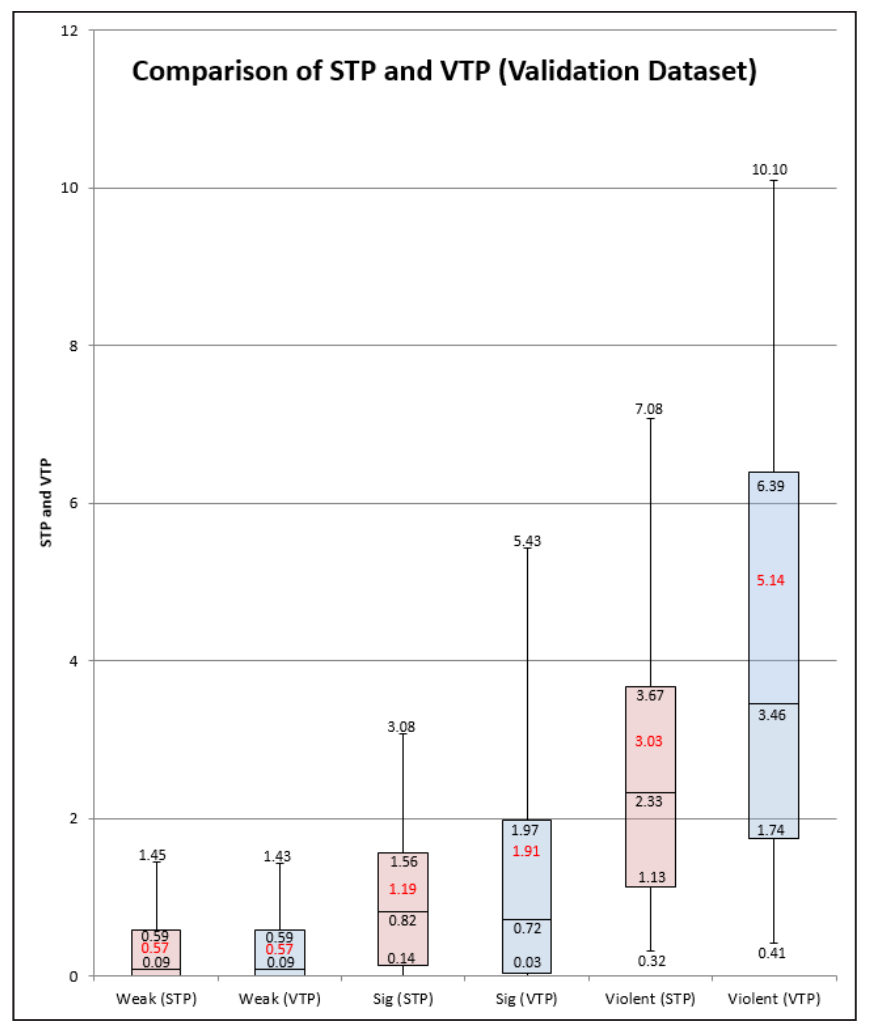

Figure 11. Same as Fig. 10 except for the validation dataset. This sample consisted of 100 weak, 100 significant, and the original 50 violent tornadoes.

and violent tornado environments. The Mann-Whitney test for the STP scored a -5.0 , and the VTP scored a -5.5. The VTP once again was the superior parameter for distinguishing violent tornado environments.

\section{Comparisons to surface objective analysis data}

The SPC surface objective analysis dataset (SFCOA; Bothwell et al. 2002) used in Smith et al. (2015) was compared to the data from this study to determine if the findings could be replicated. The SFCOA used the RUC (Benjamin et al. 2004) as its initial source from 2005 until 2012 when the Rapid Refresh (Benjamin et al. 2016) replaced it. The STP had higher values in the SFCOA dataset compared to the observed sounding dataset of this study with a mean value for violent tornadoes of 5.27 (Fig. 12) compared to the observed database of 3.03 (Fig. 10). The VTP had similar increases compared to the observational dataset between significant and violent tornadoes, with the mean VTP rising from 3.01 for significant tornadoes to 7.18 for violent tornadoes. This compares to an increase from 1.83 for significant tornadoes to 5.14 for violent 


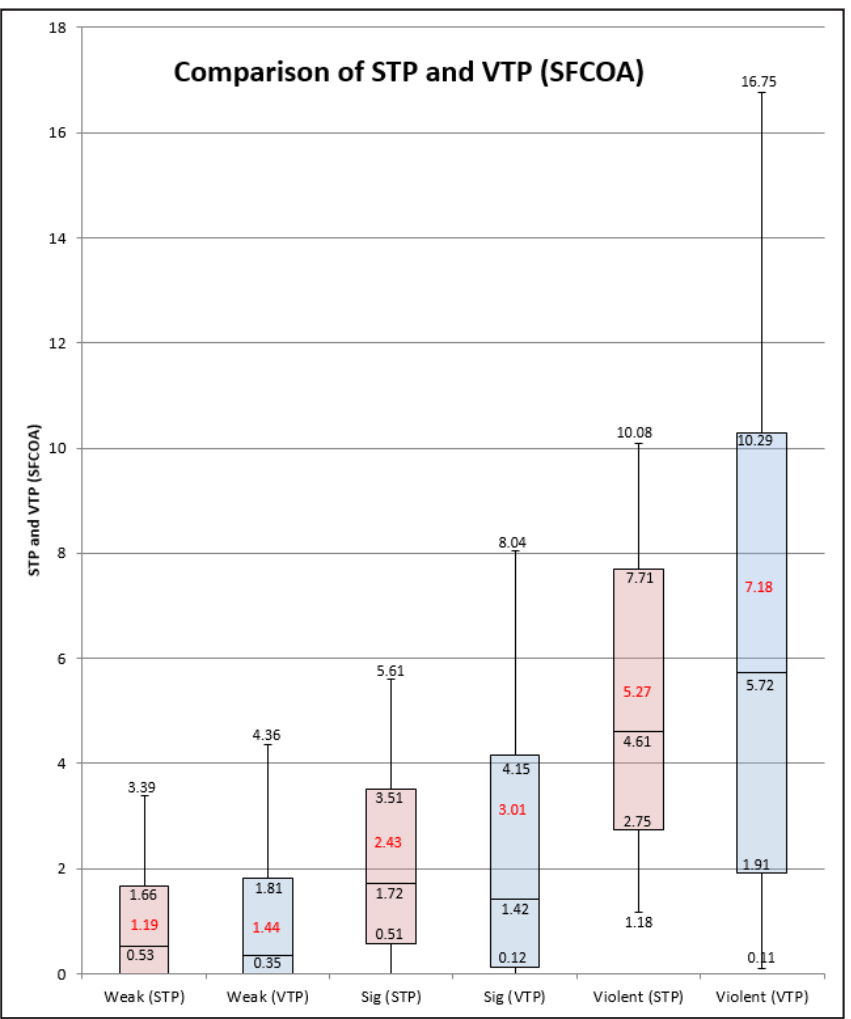

Figure 12. Same as Fig. 2 except a comparison of the STP and VTP using SFCOA data. This sample consisted of 9752 weak, 1544 significant, and 101 violent tornadoes.

tornadoes in the observed sounding database (Fig. 10). The STP and VTP values were higher overall in the SFCOA dataset primarily because ESRH values were $55 \%$ higher than those in the observed dataset. The MLCAPE, EBWD, and MLLCL were within 15\% of the observed dataset, but 3CAPE was about $30 \%$ higher and 3LR was about $20 \%$ higher in the observed dataset versus the SFCOA.

Because the SFCOA and observed soundings datasets differed significantly for some parameters, the SFCOA data were compared to the nearest grid point and time of the rawindsonde observations used in this study to test the accuracy of SFCOA data in the environments favorable for significant and violent tornadoes. The SFCOA data were available between 2003 and 2015, resulting in 17 violent and 30 significant tornado event soundings for direct comparison. The error of ESRH in the SFCOA data was less than $5 \%$ for the significant tornadoes and less than $1 \%$ for the violent tornadoes. This likely substantiates both datasets but suggests ESRH can have significant variability in time and space $(<100 \mathrm{~km})$ near tornado-producing convection owing to surface boundary interactions (Markowski et al. 1998). The higher temporal and spatial resolution of SFCOA would excel at capturing the higher values of ESRH near significant and violent tornado-producing convection. However, rawindsonde observations had $3 \mathrm{LR}$ values $0.75{ }^{\circ} \mathrm{C} \mathrm{km}^{-1}$ higher for violent tornadoes and $0.63{ }^{\circ} \mathrm{C}$ $\mathrm{km}^{-1}$ higher for significant tornadoes versus the SFCOA values. Similarly, rawindsonde observations for 3CAPE for violent and significant tornadoes averaged $8 \mathrm{~J} \mathrm{~kg}^{-1}$ higher than the SFCOA value. The lower values of low-level instability parameters from the SFCOA dataset versus the observations are likely attributed to a cool, moist bias near the surface due to a local planetary boundary layer scheme (Coniglio 2012) used in the RUC (and Rapid Refresh) and subsequently the SFCOA. Given the result of these direct comparisons, SFCOA data cannot be used independently to study the relationship of low-level instability to tornado-damage rating and should be used in an operational setting with other numerical model analyses. These errors within SFCOA likely also explain why the importance of lowlevel instability parameters had not been identified in prior research that relied upon SFCOA data.

\section{Conclusions}

Violent tornado environments were associated with greater increases within low-level instability parameters (3LR and 3CAPE) in comparison to the shear-based, LCL-based, or CIN-based components of the STP. Markowski and Richardson (2010) stated that, once a tornado is established, tilting of the surfacelayer horizontal vorticity by the extreme vertical velocity gradient associated with the tornado updraft itself probably contributes to the near-ground vertical vorticity in a significant way. In theory, higher values of low-level instability could maximize the lowlevel vertical velocities of a convective updraft and, subsequently, increase near-ground vertical vorticity. Thus, higher instability in the lower troposphere could lead to stronger tornadoes owing to the higher vertical velocities.

Prior research (Thompson et al. 2003; Grams et al. 2012) has shown that properties of the low-level wind field are crucial to not only tornadogenesis but also to intensification into the EF2 and EF3 categories. Low-level instability likely then plays an important role in further tornadic intensification into the EF4 and EF5 ranges. This study concludes that, when forecasting environments favorable for violent 
tornadoes, forecasters should closely investigate lowlevel instability to help determine the possibility of violent versus significant tornadoes. However, the near-storm environment can be highly variable on short temporal and small spatial scales (Miller 2006). Highly susceptible environments for violent tornadoes, as indicated by Miller, occur many times without a violent tornado owing to many possible failures (e.g. storm initiation, timing, convective mode, etc.). This study does not attempt to predict the occurrence of violent tornadoes but posits that the environments where violent tornadoes occur have significantly higher amounts of low-level instability than those environments that produce only significant tornadoes.

The VTP was proposed to help operational forecasters discern environments supportive of violent tornadoes. This variable is essentially the STP with an inclusion of a 3LR and 3CAPE term. The VTP had a Mann-Whitney score of -5.3 , and this is the best score compared to any variable or composite parameter researched in this study.

Acknowledgements. The authors would like to thank Jon Zeitler and John Brost for reviewing our paper. The authors also would like to thank Joe Arellano, Tom Bradshaw, Bill Bunting, and Steve Drillette for enabling us to spend time working on this project. In addition, the authors thank Gregory Patrick for proving initial insights and guidance to the study and to Matt Bunkers and Rich Thompson for providing expert opinions and suggestions during the peer-review process.

\section{REFERENCES}

Albright, S. C., and W. L. Winston, 2014: Business Analytics: Data Analysis and Decision Making. 5th ed. South-Western College Pub. 990 pp.

Ashley, W. S., 2007: Spatial and temporal analysis of tornado fatalities in the United States: 1880- 2005. Wea. Forecasting, 22, 1214-1228, Crossref.

Benjamin, S. G., and Coauthors, 2004: An hourly assimilation-forecast cycle: The RUC. Mon. Wea. Rev., 132, 495-518, Crossref.

, and Coauthors, 2016: A North American hourly assimilation and model forecast cycle: The Rapid Refresh. Mon. Wea. Rev., 144, 1669-1694, Crossref.
Bothwell, P. D., J. A. Hart, and R. L. Thompson, 2002: An integrated three-dimensional objective analysis scheme in use at the Storm Prediction Center. Preprints, 21st Conf. on Severe Local Storms, San Antonio, TX, Amer. Meteor. Soc., JP3.1. [Available online at ams.confex. com/ams/SLS WAF NWP/techprogram/paper 47482. $\underline{\mathrm{htm}}$.

Brooks, H. E., C. A. Doswell III, and J. Cooper, 1994: On the environments of tornadic and nontornadic mesocyclones. Wea. Forecasting, 9, 606-618, Crossref.

Cohen, A. E., 2010: Indices of violent tornado environments. Electronic J. Operational Meteor., 2010-EJ6, 1-24. [Available online at http://nwafiles.nwas.org/ej/ pdf/2010-EJ6.pdf.]

Coniglio, M. C., 2012: Verification of RUC 0-1-h forecasts and SPC mesoscale analyses using VORTEX2 soundings. Wea. Forecasting, 27, 667-683, Crossref.

Craven, J. P., and H. E. Brooks, 2004: Baseline climatology of sounding derived parameters associated with deep moist convection. Natl. Wea. Dig., 28, 13-24. [Available online at www.nssl.noaa.gov/users/brooks/public html/ papers/cravenbrooksnwa.pdf.]

Edwards, R., A. R. Dean, R. L. Thompson, and B. T. Smith, 2012: Convective modes for significant severe thunderstorms in the contiguous United States. Part III: Tropical cyclone tornadoes. Wea. Forecasting, 27, 15071519 , Crossref.

Davies, J. M., 2002: Significant tornadoes in environments with relatively weak shear. Preprints, 21st Conf. on Severe Local Storms, San Antonio, TX, Amer. Meteor. Soc., 16.5. [Available online at ams.confex.com/ams/ pdfpapers/46965.pdf.]

, 2006: Tornadoes in environments with helicity and/or high LCL heights. Wea. Forecasting, 21, 579-594, Crossref.

Evans, J. S., and C. A. Doswell III, 2001: Examination of derecho environments using proximity soundings. Wea. Forecasting, 16, 329-342, Crossref.

Fuhrmann, C. M., C. E. Konrad II, M. M. Kovach, J. T. McLeod, W. G. Schmitz, and P. G. Dixon, 2014: Ranking of tornado outbreaks across the United States and their climatological characteristics. Wea. Forecasting, 29, 684-701, Crossref.

Grams, J. S., R. L. Thompson, D. V. Snively, J. A. Prentice, G. M. Hodges, and L. J. Reames, 2012: A climatology and comparison of parameters for significant tornado events in the United States. Wea. Forecasting, 27, 106 123, Crossref.

Knupp, K. R., and Coauthors, 2014: Meteorological overview of the devastating 27 April 2011 tornado outbreak. Bull. Amer. Meteor. Soc., 95, 1041-1062, Crossref.

Markowski, P. M., J. M. Straka, E. N. Rasmussen, and D. O. Blanchard, 1998: Variability of storm-relative helicity during VORTEX. Mon. Wea. Rev., 126, 2959-2971, Crossref. 
, and Y. Richardson, 2010: Mesoscale Meteorology in Midlatitudes. Wiley, $430 \mathrm{pp}$.

McCarthy, D. W., and J. Schaefer, 2004: Tornado trends over the past thirty years. Preprints, 14th Conf. on Applied Climatology, Seattle WA, Amer. Meteor. Soc., 3.4. [Available online at ams.confex.com/ams/84Annual/ techprogram/paper 72089.htm.]

Miller, D. J., 2006: Observations of low level thermodynamic and wind shear profiles on significant tornado days. Preprints, 23rd Conf. on Severe Local Storms, St. Louis, MO, Amer. Meteor. Soc., 3.1. [Available online at ams.confex.com/ams/pdfpapers/115403.pdf.]

Nowotarski, C. J., P. M. Markowski, and Y. P. Richardson, 2011: The characteristics of numerically simulated supercell storms situated over statically stable boundary layers. Mon. Wea. Rev., 139, 3139-3162, Crossref.

Potvin, C. K., K. L. Elmore, and S. J. Weiss, 2010: Assessing the impacts of proximity sounding criteria on the climatology of significant tornado environments. Wea. Forecasting, 25, 921-930, Crossref.

Rasmussen, E. N., and D. O. Blanchard, 1998: A baseline climatology of sounding-derived supercell and tornado forecast parameters. Wea. Forecasting, 13, 1148-1164, Crossref.

Smith, B. T., R. L. Thompson, A. R. Dean, and P. T. Marsh, 2015: Diagnosing the conditional probability of tornado damage rating using environmental and radar attributes. Wea Forecasting, 30, 914-932, Crossref.

Thompson, R. L., R. Edwards, J. A. Hart, K. L. Elmore, and P. M. Markowski, 2003: Close proximity soundings within supercell environments obtained from the Rapid Update Cycle. Wea. Forecasting, 18, 1243-1261, Crossref.

, B. T. Smith, J. S. Grams, A. R. Dean, and C. Broyles, 2012: Convective modes for significant severe thunderstorms in the continuous United States. Part II: Supercell and QLCS tornado environments. Wea. Forecasting, 27, 1136-1154, Crossref.

Togstad, W. E., J. M. Davies, S. J. Corfidi, D. R. Bright, and A. R. Dean, 2011: Conditional probability estimation for significant tornadoes based on Rapid Update Cycle (RUC) profiles. Wea. Forecasting, 26, 729-743, Crossref. 